\title{
1 Prediction of drug-protein interaction and drug repositioning using machine
}

\section{2 learning model}

3 Yu-Ting $\operatorname{Lin}^{1}$, Sheh-Yi Sheu ${ }^{1,2^{*}}$, Chen-Ching $\operatorname{Lin}^{1 *}$

4

$5 \quad{ }^{1}$ Institute of Biomedical Informatics, National Yang-Ming University, Taipei, Taiwan

$6 \quad{ }^{2}$ Department of Life Science and Institute of Genome Science, National Yang-Ming

7 University, Taipei, Taiwan

$8 \quad$ * Corresponding authors

9

10 Abstract

\section{Background}

12 Traditional drug development is time-consuming and expensive, while

13 computer-aided drug repositioning can improve efficiency and productivity. In this

14 study, we proposed a machine learning pipeline to predict the binding interaction

15 between proteins and marketed or studied drugs. We then extended the predicted

16 interactions to construct a protein network that could be applied to discover the

17 potentially shared drugs between proteins and thus predict drug repositioning. 


\section{Methods}

2 Binding information between proteins and drugs from the Binding Database and the

3 physicochemical properties of drugs from the ChEMBL database were used to build

4 the machine learning models, i.e. support vector regression. We further measured

5 proportionalities between proteins by the predicted binding affinity and introduced

6 edge betweenness centrality to construct a protein similarity network for drug

7 repositioning.

\section{Results}

9 As the proof of concept, we demonstrated our machine learning approach is capable

10 of reflecting the binding strength between drugs and the target protein. When

11 comparing coefficients of protein models, we found proteins SYUA and TAU that

12 may share common ligand which were not in our training data. Using the edge

13 betweenness centrality network based on the prediction proportionality of protein

14 models, we found a potential target, $\mathrm{AK} 1 \mathrm{C} 2$, of aspirin and of which the binding

15 interaction had been validated.

\section{Conclusions}

17 Our study could not only be applied to drug repositioning by comparing protein

18 models or searching the protein-protein network, but also to predict the binding 
1 strength once the sufficient experimental data was provided to train the protein

2 models.

3 Keywords: Drug repositioning, Machine learning, Protein network

4

\section{$5 \quad$ Background}

6 In pharmacology, drugs can be classified into several classes by their sizes,

7 mechanisms of action, chemical structures or other different criteria. Among these

8 classes, a small molecule drugs (SMD) often refers to an organic compound with low

9 molecular weight (<900 dalton) and small size. Compared to biological drugs, SMDs

10 have simple and well-defined structures, leading to easier synthesis and a more

11 predictable chemical process in manufacturing and characterizing. In general, the

12 development of one SMD is mainly processed via 5 stages: I. Discovery and

13 Development, II. Preclinical Research, III. Clinical Research, IV. FDA Review, and V.

14 FDA Post-Market Safety Monitoring, to make sure that the new pharmaceutical

15 compounds are safe to be distributed to the market $[1,2]$. Among the cycle of drug

16 development, the first step, drug discovery, is one of those that cost the most, up to

17674 US\$ millions per one approved drug and taking 2 5 years in average [3-5]. To

18 boost the process, novel techniques were proposed in recent years, like combinatorial

19 chemistry methods for compound formulation as well as using virtual screening and 
1 high-throughput screening to filter out candidate drugs. By using a computational,

2 data-based, or robotic method, the accuracy and efficiency of drug discovery were

3 highly improved. Also, drug repositioning plays an important role in accelerating drug

4 development as well.

5 Drug repositioning (a.k.a. drug repurposing) has increasingly become an essential

6 strategy in drug development by reinvestigating existing de-risked and approved

7 drugs for new therapeutic purposes [6]. Despite some remaining technological and

8 regulatory challenges needed to be solved, this novel strategy is more efficient,

9 economical, and riskless. On the other hand, with a great amount of biochemical,

10 pharmacological, and clinic data collected in these decades, there have been many

11 emerging techniques like machine learning and network analysis that can take

12 advantage of big data and promote efficiency and productivity [7].

13 So far, studies using machine learning approaches for drug repositioning have

14 become more and more popular [8-10]. Napolitano F, et al. ( $J$ Cheminform, 5, 30,

15 2013) proposed a method based on an SVM (support vector machine) classifier to

16 predict the corresponding disease classes of drugs [11]. Kim E, et al. (BMC

17 Bioinformatics, 20, 247, 2019) constructed models using multiple classification

18 algorithms, including logistic regression, random forest, and SVM to predict potential

19 indications for existing drugs [12]. The method developed by Zhanchao Li (Anal. 
1 Methods, 10, 4152-4161, 2018) uses the random forest algorithm to predict the

2 binding affinity between a compound and a protein directly. Based on the graph

3 theory, the model uses compound-compound similarity, protein-protein interactions,

4 and compound-protein interaction to characterize the binding interaction and may be

5 applied to high-throughput virtual screening [13].

6 In this study, we employed the support vector regression (SVR) algorithm to build

7 models to predict the binding interaction between proteins and drugs. The inhibition

8 constant $\left(\mathrm{K}_{\mathrm{i}}\right)$, which represented the binding strength, was used as label; and

9 structural fingerprint format and physicochemical properties of drugs were used as

10 features. Consequently, we selected 556 protein models to forecast the binding

11 strength with 86,475 drugs. Additionally, we adapt the similarity of predicted binding

12 strength to drugs between proteins to construct a protein similarity network.

13 Furthermore, we proposed an auxiliary network constructed from edge betweenness

14 centralities (eBC) to discover the potential drug repositioning between proteins. With

15 the aid of the network, we found a potential target protein AK1C2 of aspirin by its

16 known target $\mathrm{PGH} 1$ and the binding interaction had been reported, providing a

17 successful application of our method for drug repositioning. Briefly, our method can

18 not only be applied to drug repositioning by comparing protein models or searching 
1 the protein-protein network, but also by predicting the binding strength directly once

2 the experimental data are sufficient to train the protein models.

\section{Methods}

\section{Information of proteins and drugs}

5 To predict the strengths of unknown binding interactions of proteins and drugs, we

6 needed experimental data of known protein-drug interactions train the machine

7 learning model. In this study, the protein-drug interaction data were downloaded from

8 the Binding Database [14-18] and interactions with non-human proteins were

9 discarded. Next, the drug compounds are provided with the structural information,

10 PubChem CID, InChIKey, and the binding strength to the target protein, which is

11 inhibition constant in this study, were recognized as informative and kept. For

12 proteins, only human proteins with specific UniProt entry names were adopted.

13 Among the information, the binding strength is the label that our machine learning

14 model attempts to predict; and structural information is one part of the feature in the

15 model. Noted that in our study, only drug information was used as features to describe

16 the binding interactions in each protein model. The circumstances of multiple binding

17 sites of a protein were not considered and only the data with the highest value of

18 binding strength was kept if there were more than one data of a drug-protein pair. 
1 The structures of drugs were described as "Molecular ACCess System (MACCS)

2 keys" [19-21] that consists of a list of binary values (0 or 1) and contains 166 key

3 descriptors to encode a structure. The MACCS keys of drugs were used as a 166-bit

4 feature to train the machine learning models.

5 On the other hand, since the property data describing physicochemical

6 characteristics of a drug could affect the binding between drug and target protein, we

7 further add them as features in our model. The physicochemical properties of drug

8 compounds were obtained from the ChEMBL database. We used the Python library

9 “chembl_webresource_client", which is officially developed and supported by the

10 ChEMBL group and selected 17 properties that might have relevance with binding

11 and thus help in prediction. The properties are introduced following their names used

12 in the library (Table S1). Finally, the 17 physicochemical properties were combined

13 with the 166-bit structural fingerprint to form 183 features that were used in our

14 machine learning model.

\section{Binding strength of drug-protein interactions}

16 In this study, we used $-\log K_{i}$ as label to represent the binding strength of a

17 drug-protein interaction and to train the protein models. In fact, the Gibbs Free

18 Energy $\left(\Delta G^{0}\right)$ is also frequently used to represent the binding affinity of a drug and

19 target. From the aspect of thermodynamics, the lower the $\Delta \mathrm{G}^{0}$ is, the stronger the 
1 interaction is. And $\Delta \mathrm{G}^{0}$ can be calculated by an equilibrium constant $\left(K_{e q}\right)$ as

$2 \Delta G^{0}=-R T \ln K_{e q}$, where $R$ is the ideal gas constant that equals $8.314 \mathrm{~J} \mathrm{~mol}^{-1} \mathrm{~K}^{-1}$

3 and $\mathrm{T}$ is the temperature on the Kelvin scale [22]. The equilibrium constant is the

4 dissociation constant in this case, then the $\Delta \mathrm{G}^{0}$ can be further represented by $K_{i}$ as

$5 \Delta G^{0}=-R T \ln \frac{1}{K_{i}}$. Because $R$ and $T$ are both constants in the equilibrium, we can

6 infer that $\Delta \mathrm{G}^{0}$ is positively proportional to $\log K_{i}$ by multiplying $\ln K_{i}$ by a constant

$7 \log e$. For the reasons mentioned above, we chose $\log K_{i}$ to represent the strength of

8 the binding of a drug-protein interaction.

\section{Construction of protein models}

10 In this study, we aim to develop a machine learning model to predict the binding

11 affinity of a drug to a specific protein. The machine learning algorithm we chose here

12 is support vector regression (SVR), which is a regression version of support vector

13 machine (SVM) and works by finding a hyperplane that can minimize the distance

14 from the hyperplane to the instance and cover as most data points as possible in the

15 same time [23]. Because the model of SVM or SVR can be recognized as a weighted

16 sum of the support vectors, it has advantages of reduced computational time and

17 storage requirements over other frameworks. And the principle gives SVM or SVR

18 good generalization capability and better robustness over those algorithms that

19 involve randomness elements. 
1 To train our prediction model, we obtained 152,112 binding information among

21,042 proteins and 86,475 drugs from the BindingDB (Dataset S1) [14-18]. The drug

3 in each data was described by 183 features which consisted of a 166-bit MACCS

4 structure fingerprint [19-21] and 17 physicochemical properties (Table S1) from the

5 ChEMBL database (Dataset S2) [24, 25]. Here we introduced inhibition constant $\left(K_{i}\right)$

6 to represent the binding strength of a drug with its target protein. $K_{i}$ is defined as

$7 \quad K_{i}=\frac{[E][I]}{[E I]}$ where $[\mathrm{E}],[\mathrm{I}]$, and $[\mathrm{EI}]$ are the equilibrium concentrations of enzyme,

8 inhibitor, and the complex [26]. The smaller the $K_{i}$ value, the greater the binding

9 strength of the inhibitor with its target, and the better the potency of the drug in most

10 cases. Since the $K_{i}$ value can quantitatively reflect how tightly a compound binds to a

11 particular protein, we used it to represent the binding strength of a compound-protein

12 interaction. In our data, however, the experimental $K_{i}$ values show an exponential

13 distribution and many of the experimental $K_{i}$ values are larger than 1,000 while some

14 of the others are less than one. Given that many regression algorithms are based on

15 the concept of least squares method to approximate data, the large difference in scale

16 between observations leads to poor fitting since the residues on the larger scale weigh

17 moreover those on a smaller scale. Therefore, we transformed the experimental $K_{i}$

18 values into logarithmic ones, to offer the machine learning model a dataset with a

19 linear distribution. Furthermore, to make the study results easier to be comprehended, 
1 we used $-\log \mathrm{K}_{\mathrm{i}}$ as the indicator of binding affinity and the label of our machine

2 learning model, while the larger the value is, the stronger the compound-protein

3 interaction is.

4 Before model training, each property feature among samples were standardized

5 by computing the standard score $\mathrm{z}$ as $z=(x-\mu) / \sigma$, where $\mu$ is the mean and $\sigma$ is

6 the standard deviation of the feature throughout all samples. We then built a

7 prediction model for each protein using only the dataset targeting it. The model

8 trainings were all default settings except the parameter "kernel" was specified to be

9 "linear". When training the SVR model, we randomly split the dataset into two groups:

$1070 \%$ of the dataset as the training set to build the model and evaluate the prediction

11 accuracy, and the other $30 \%$ as the testing set for validation to assess how the model

12 would generalize to an independent dataset. Then the models applied for further

13 analysis would be trained by their complete datasets.

14 The evaluation we used here to see how well a model could predict was the

15 Spearman correlation coefficient (SCC, $\rho$ ), which is a nonparametric measure of rank

16 correlation to assess the monotonic relationships [27-29]. When comparing the

17 accuracies among models, we introduced the Fisher transformation to take the data

18 number into account [30]. The transformation of the sample SCC value $r$ can be

19 computed by 


$$
F(r)=\frac{1}{2} \ln \left(\frac{1+r}{1-r}\right)
$$

2 When given the sample size $n$, the $z$-score of $r$ is

$$
z=\sqrt{\frac{n-3}{1.6}} F(r)
$$

4 which approximately follows a standard normal distribution under the null hypothesis

5 of statistical independence $(\rho=0)$.

\section{Construction of the protein network for drug repositioning}

7 Having the predictions of binding strength, the protein similarity was calculated by

8 proportionality [31-33]. However, since the protein similarity only considers the

9 direct association between them, we further constructed an auxiliary network which

10 was based on proportionalities and was capable of detecting the indirect similarity

11 between proteins.

12 To keep the highly confident protein pairs, we first removed the protein pairs

13 with low proportionality, which is the majority of the network, and applied the

14 concept of the shortest paths in the network to consider the indirect correlations since

15 the two adjacency proteins might not have the strongest correlation when computing

16 direct similarity.

17 The concept of the shortest path was applied in our study by betweenness

18 centrality, which is used to evaluate the importance of nodes of edges in a network. If

19 a network is weighted, the shortest path is the one having the maximal sum of the 
1 weights of the edges. The edge betweenness centrality (eBC) of an edge is defined by

2 the number of these shortest paths that pass through this edge and the $\mathrm{eBC}$ of an edge

$3 v$ can be computed as

$$
g(v)=\sum_{s \neq v \neq t} \frac{\sigma_{s t}(v)}{\sigma_{s t}}
$$

4 where $\sigma_{\text {st }}$ is the sum of the shortest path from node s to node t, and $\sigma_{s t}(v)$ is the

5 number of the paths that pass through $v$ [34].

6 For our original network of proportionality, we calculated the eBC of every edge

7 in it and removed those whose $\mathrm{eBC}$ was 0 . Then each remained edge was assigned a

8 new weight by its $\mathrm{eBC}$ value for next $\mathrm{eBC}$ calculation. The cycle of $\mathrm{eBC}$ calculation,

9 removing edges, and assignment of new weights was repeated until convergence

10 (Figure S1A). Here we used the mean-square error (MSE) between the edge weights

11 and the eBC values in a cycle to tell whether the calculation was converged. The

12 number of remained edges maintained the same after the $15^{\text {th }}$ cycle and so did the

13 MSE after the $18^{\text {th }}$ (Figure S1B). We then used the eBC values calculated in the $18^{\text {th }}$

14 cycle to build an eBC-based protein-protein network for further analysis.

\section{Results}

\section{Proof of concept for the machine learning model}

17 To determine the capability of our machine learning pipeline in predicting the binding

18 affinity of a drug to a specific protein, we first used the models trained from AA2AR, 
1 DHI1, and AL5AP separately as a proof of concept. First, we partitioned our dataset

2 into training and testing sets to perform cross-validation for these three proteins. We

3 observed that the experimental $-\log K_{i}$ values were strongly and positively correlated

4 with the predicted $-\log K_{i}$ values from training and testing sets (Table 1 and Figure 1a).

5 These results implicated that this approach might be capable of reflecting the binding

6 strength of a drug to target protein well to some extent. However, the process of

7 random sampling for cross-validation makes deciding the best model uncertain.

8 Additionally, to get the best performance when given an unknown drug, the complete

9 dataset targeting a specific protein should be used to train the model. Hence the

10 accuracy and the risk of overfitting using the complete dataset to train and test the

11 model were examined (Figure 1a). The SCC values of complete datasets in the three

12 models were close to the distributions of the training sets as well as testing sets (Table

13 1), demonstrating the low risk of overfitting from complete dataset. Though the SCC

14 values themselves may be affected by the number of drugs, leading to lower

15 accuracies of the complete datasets than the training sets, the models built by

16 complete datasets were still representative for predictions. Thus, the models in the

17 following study were trained by their own complete dataset for best predictions.

18 Table 1. Performance of three models: AA2AR, DHI1, and AL5AP.

\begin{tabular}{|c|c|c|c|}
\hline Model & AA $2 A R$ & DHI1 & AL5AP \\
\hline & Training & Training & Training \\
\hline
\end{tabular}




\begin{tabular}{c|cc|cc|cc}
\hline Number of Drugs & \multicolumn{2}{|c|}{3452} & \multicolumn{2}{c|}{259} & \multicolumn{2}{c}{1411} \\
Avg. SCC & 0.72 & 0.68 & 0.91 & 0.68 & 0.7 & 0.62 \\
Range of SCC & $0.7 \sim 0.74$ & $0.63 \sim 0.73$ & $0.87 \sim 0.95$ & $0.42 \sim 0.84$ & $0.67 \sim 0.73$ & $0.53 \sim 0.71$ \\
SCC of complete dataset & \multicolumn{2}{|c|}{0.71} & \multicolumn{2}{|c|}{0.89} & \multicolumn{2}{|c}{0.69} \\
\hline
\end{tabular}

1

2 We further examined the ability of a protein model to select the drugs that would

3 have binding interaction with the corresponding protein from all the drugs in the

4 dataset. For example, the $-\log K_{i}$ values of all 86,475 drugs predicted from the AA2AR

5 model were compared with that of AA2AR experimentally-validated drugs. We found

6 that the $-\log K_{i}$ values distribution of experimentally validated drugs were significantly

7 higher than those of other drugs (Figure 1b). Additionally, the experimentally

8 validated drugs were observed to be significantly enriched in the drugs with higher

9 predicted $-\log K i$, and the amount decreased over the rankings. (Figure 1b). This

10 supported that our model could not only pick out the drugs that were already known

11 to have binding interaction with the modeled protein but also implicate the

12 unvalidated drugs in the top rankings may have potential binding ability to the

13 modeled protein. To further demonstrate that the models could predict the drugs

14 strongly binding with the modeled protein, we investigated the drugs with high

15 predicted and experimental $\log K_{i}$ to AA2AR. The compound of

16 3-[2-[4-[2-Fluoro-4-(2-methoxyethoxy)phenyl]piperazin-1-yl]ethyl]-8-(furan-2-yl)-[1,

$172,4]$ triazolo[5,1-f]purin-5-amine is predicted with the secondly highest $-\log K_{i}$ value of 
10.094 among the drugs targeting AA2AR and possessed the $99^{\text {th }}$ high

2 experimental $-\log K_{i}$ value of 0.097 in the training set. The experimental $k_{i}$ value of

3 the compound binding to $\mathrm{AA} 2 \mathrm{AR}$ is $0.8 \mathrm{nM}$, making the compound a promising

4 antagonist to AA2AR. The possible application of the drug could prevent motor

5 disturbances caused by defects related to dopamine D2 receptors and further serve as

6 an anti-symptomatic drug for the treatment of Parkinson's disease [35]. Briefly, using

7 AA2AR, DHI1, and AL5AP models as exampling proof of concept showed that our

8 method based on the machine learning approach could be used to predict the relative

9 binding strength between a drug and a protein, which could be applied to the

10 screening of candidate drugs in drug development.

\section{Performance of the machine learning model}

12 After demonstration of the proof of concept for our machine learning pipeline, we

13 then attempted to construct the prediction models for all the proteins in the database.

14 However, the machine learning approaches were known to be affected by the sample

15 size - number of experimentally validated drugs in this study. We also observed that,

16 compared to AA2AR and AL5AP model, DHI1 model, which possessed fewer

17 experimentally validated drugs, showed larger difference of SCC between training

18 and testing set (Figure S2). Therefore, we verified the effect of the training data size

19 on the performances of the prediction models. Among the total 1,042 proteins from 
1 the BindingDB, over $46 \%$ of them have less than 10 data of binding information with

2 drugs and only $21 \%$ with more than 100 data (Figure 2a). Furthermore, we found that

3 as the number of drugs increased, the SCC z-score values of model predictions

4 became higher, suggesting better accuracies (Figure 2b). Also, the more

5 experimentally-validated drug data a protein model was built by, the smaller the

6 standard deviation of the SCC distribution (Figure 2c) and the more unapparent the

7 SCC difference between the model trained by the complete dataset and by the training

8 set (70\% of complete dataset) were observed (Figure $2 \mathrm{~d}$ ). These results also indicated

9 that the model trained for the protein with more experimentally validated drugs could

10 be more stable. Accordingly, to prevent models from overfitting and inaccuracies

11 caused by lacking training data, 556 proteins (53\% of all) with more than 10 drug data

12 were retained for the following analysis. Among 556 proteins, 513 (92.26\%) protein

13 models are provided with SCC z-score greater than two, suggesting the high accuracy

14 of the machine learning pipeline in predicting relative $-\log K i$ between drugs and

15 proteins.

16 Next, we testified the model specificities by using the datasets from the other

17 proteins. For example, to test the AA2AR model, we used it to predict the $-\log K_{i}$ of

18 data originally targeting DHI1 and AL5AP. Then, for DHI1 and AL5AP, we compared

19 their $-\log K_{i}$ predicted by AA2AR model to their experimental $-\log K_{i}$ separately. As 
1 expected, the SCC between experimental $-\log K_{i}$ targeting DHI1 and predicted $-\log K_{i}$

2 from AA2AR model was -0.21 ; and the SCC between experimental $-\log K_{i}$ targeting

3 AL5AP and predicted $-\log K_{i}$ from AA2AR model was 0.09 (Figure S3). The two

4 results suggested that the AA2AR model could not be used to predict the $-\log K_{i}$ of

5 drugs binding to DHI1, neither AL5AP. Same evaluation was performed on DHI1 and

6 AL5AP models and it was alluded that a protein model could only be applied to

7 predict the drugs targeting the protein itself and showing the specificity of the

8 prediction model (Figure S3). The investigation of all the models was further

9 evaluated by the distribution of the average z-score. For all 556 proteins, every model

10 was used to predict the $-\log \mathrm{K}_{\mathrm{i}}$ of other 555 sets of drugs and their target proteins, and

11 the average z-score value was recorded (Figure $2 \mathrm{e}$ ). Notably, only $1.6 \%$ proteins are

12 provided with average $\mathrm{z}$-score greater than two, showing that most of the models were

13 unsuitable to predict the binding interaction targeting proteins other than themselves

14 and assuring the overall specificities of models.

15 Nevertheless, based on the reason, the specificities of models indicated that if a

16 model could predict the drugs of another protein well, there might be some

17 similarities or shared characteristics between the two proteins that could be applied to

18 drug repositioning. For example, the model of protein DRD2 was trained by 5,155

19 drug data and had a z-score of 20 when predicting the 844 drugs binding to their 
1 experimental target protein BCL2. The two proteins shared significantly enriched GO

2 biological process ( $\mathrm{p}$-value $<0.0001$, Fisher's exact test), showing their functional

3 similarity. DRD2 is dopamine D2 receptor whose activity is mediated by G proteins

4 and is the main receptor for most antipsychotic drugs [36]. BCL2 is apoptosis

5 regulator (B-cell lymphoma 2) which can suppress or induce apoptosis and regulate

6 cell death by controlling the mitochondrial membrane permeability [37, 38]. The

7 relevance of the two proteins in several signaling pathways has been studied. In

8 non-small cell lung cancer, it was found that the upregulation of BCL2 is related to

9 the knockdown of DRD2 and the overexpression of the DRD2 gene may decrease the

10 mRNA level of BCL2 [39]. In another research on Parkinson's disease, it was found

11 that the inhibition of hsa-miR-200a can upregulate BCL2 and DRD2 via the

12 cAMP/PKA signaling pathway and suppress apoptosis in striatal neuron cells [40].

13 Though no research reported the shared drugs of these proteins, we still demonstrated

14 that using model specificity could help find proteins with similar biological roles and

15 the relevance of them may provide information for medication.

\section{Model comparison and drug repositioning}

17 We have demonstrated that the SVR models could be able to predict the

18 relative $-\log K_{i}$ value between a drug and a target protein. Therefore, our approach

19 could be a feasible application in compound screening during drug development, 
1 especially for repositioning of the approved drugs that are provided with detail and

2 complete experimental information. For example, two proteins with similar models

3 after training might possess similar drug binding characteristics. Accordingly, their

4 models might be able to predict promising drugs as a potential treatment for each

5 other, a.k.a. drug repositioning. We then calculated the SCC of coefficients between

6 models to assess the similarity of drug-binding profiles between proteins. That is, two

7 proteins with higher SCC are more similar to each other on drug-binding

8 characteristics. Accordingly, for each model pair, we inspected the association

9 between the SCC of model coefficients and the average drug number (Figure 3). The

10 results showed that when the models were trained by few drugs, their model

11 similarities varied from 0.89 to -0.56 and when trained by more drugs, the model

12 similarities became more convergent and lied mostly between \pm 0.2 The $p$-values

13 were examined and the smallest positive SCC value with significance $(p$ value $<0.05)$

14 was 0.15 (the green line in Figure 3). The proteins of the model pairs above the

15 significance line could be worth to be investigated for drug repositioning. Among all

16 the protein pairs, three of them are with more than 3,000 drugs in average and SCC

17 higher than 0.3. In two of the pairs, CAH1-CAH2 and DRD2-DRD3 were from the

18 same family. Since proteins in a family typically have similar structures, functions,

19 and sequence similarity, it was reasonable for their prediction models with similar 
1 feature weight ranking, that is, with high SCC. This also demonstrated that our

2 method could recognize the proteins with some shared characteristics. However, for

3 the proteins in the same family, most of the experimented drugs were the same,

4 limiting the efficacy of repositioning. Thus, we focused on another protein pair,

5 5HT2A-DRD3. Protein 5HT2A is a G-protein coupled receptor for

6 5-hydroxytryptamine (serotonin) and can affect neural activity, perception, cognition,

7 and mood. It also plays roles in the regulation of behavior, intestinal smooth muscle

8 contraction, and arterial vasoconstriction [41, 42]. DRD3 is a dopamine receptor

9 whose activity is mediated by $\mathrm{G}$ proteins which inhibit adenylyl cyclase and can

10 promote cell proliferation [43]. Among 2,683 drugs of 5HT2A and 3,535 drugs of

11 DRD3, 914 shared drugs are significantly over-represented (p-value $<0.0001$,

12 Fisher's exact test), suggesting that they did share binding interaction that had been

13 under study.

14 However, for the models trained by few drugs, their comparisons may not be

15 convincing enough with a wide range of model coefficients similarities since it was

16 demonstrated that the models performed better with more drugs. Thus, once there

17 were models that were trained by enough data and had high correlations, the two

18 proteins may have alike binding interactions. Another problem was that the difference

19 in the sample size of models may lead to deviations when comparing the coefficients 
1 directly, making it inappropriate to build a network by model similarities. Therefore,

2 we proposed the construction of a protein-protein network based on prediction

3 similarity between all protein models to reduce the data amount effect on a single

4 model and provide insights on the mutual connection of proteins.

\section{$5 \quad$ Drug repositioning using the $\mathrm{eBC}$ network}

6 To apply the protein models to drug repositioning from an integral aspect, we

7 constructed protein similarity networks guided by the SVR model (Figure 4a and 4b).

8 In the network, edges linking proteins are weighted by the proportionality calculated

9 from the predicted $-\log K i$. To note, the distances between proteins in the network were

10 assigned by 1 - proportionality. In other words, edges with higher proportionality, i.e.

11 more similar, were assigned by shorter distance. Next, we used the edge betweenness

12 centrality (eBC) to identify the shortest (highest similarity) paths between proteins.

13 Accordingly, the indirect association between proteins, that is, the paths with highest

14 similarity included more than one proteins, could be discovered. Interestingly, the

15 average GO term similarity of protein pairs with a two-step distance was the highest

16 (Figure 4c), meaning that when computing similarities via one intermediate protein

17 allows the $\mathrm{eBC}$ network to represent the correlation of the roles that the proteins play

18 in biological processes. Hence it may be feasible for drug repositioning to consider 
1 the proteins' second neighbors in the eBC network if they would have shared drugs

2 and binding interactions.

3 To determine the reliability of our eBC network for drug repositioning, we chose

4 aspirin (acetylsalicylic acid) as an example to find its potential target protein. Aspirin

5 is a drug widely used to reduce pain, fever, or inflammation, and also applied to

6 prevent strokes and blood clots. One of the known target proteins of aspirin is

7 cyclooxygenase-1 (COX-1), which will be irreversibly inhibited by the acetylation of

8 a serine residue in the active site [44-46]. Basing on COX-1, we then tried to find

9 proteins in the eBC network that may also be targets of aspirin. Among the proteins

10 that were in two-step distance from COX-1, AK1C2 had the highest 2-step GO term

11 similarity with a value of 0.67 . AK1C2 is aldo-keto reductase family 1 member $\mathrm{C} 2$

12 that is encoded by gene $A K R I C 2$. The enzyme catalyzes the inactivation of

14 5-alpha-androstane-3-alpha,17-beta-diol (3-alpha-diol) [47, 48]. The selective loss of

15 AK1C2 may have the relevance of breast and prostate cancers $[49,50]$ and the

16 overexpression of it may be a high-risk factor in bladder cancer [51]. In our dataset

17 used to train the AK1C2 model, none of the data was about the binding of aspirin to

18 AK1C2, leaving aside the possibility that the network had already had the information

19 of this binding interaction. In a paper studying the inhibition of AK1C2 by 
1 nonsteroidal anti-inflammatory drug (NSAID) analogs proposed in 2009 by Michael

2 C. Byrns et al., it was reported that $\mathrm{IC}_{50}$ value of inhibition of $\mathrm{AK} 1 \mathrm{C} 2$ by aspirin was

$316 \mu \mathrm{M}$. Also according to the study, the concentrations of aspirin and its deacetylated

4 product salicylic acid needed for inhibition were lower than those of other NSAIDs 5 [52].

6 The GO term similarity of biological process between COX-1 (PGH1) and

7 AK1C2 in our network was calculated via EST2, a cocaine esterase. Most of their

8 shared GO biological process terms were related to the metabolic process for

9 substances including lipid, organic acid, carboxylic acid, and others. The other terms

10 were mainly related to cellular responses to stimulus. Though without support from

11 known experimental information, it might be possible that there were some

12 correlations between the shared drug and common GO biological process terms of the

13 two proteins, which was worthy of further studying.

\section{Discussion}

15 We have proposed a machine learning pipeline to discover the potential drug

16 positioning for proteins. To further understand which structure characteristics of drugs

17 affecting the binding with proteins, we analyzed the feature importance of the learning

18 functions. Here we chose three models with the highest SCC $z$-score values of

19 predictions and inspected the absolute values of weights of their physicochemical 
1 property features. The protein model with the highest $z$-score of 52.2 among all the

2 models was trained from FA10 by 3,158 binding data. Among 17 physicochemical

3 property features in the FA10 model, the absolute values of their coefficients ranged

4 from 0.05 to 0.89 . The feature with the largest absolute weight of -0.89 was the

5 number of non-hydrogen atoms in the molecule, accounted for $10.5 \%$ of the 17

6 features. The one with the second-high absolute value of weight was the polar surface

7 area $(0.84,9.9 \%$ of all); the third feature was the molecular weight of the parent form

8 of the molecule $(0.83,9.9 \%$ of all). The protein model with the second-high

9 prediction z-score of 50.89 was AA2AR trained by 3,452 data. The absolute values of

10 its features' coefficients ranged from 0.01 to 1.09 . The three top features in

11 descending order of their absolute values of weights were the number of

12 non-hydrogen atoms in the molecule $(1.09,22.3 \%$ of all), the count of hydrogens

13 attached to nitrogen or oxygen atoms $(0.79,16.2 \%$ of all), and the number of

14 hydrogen bond donors $(0.48,9.9 \%$ of all). The third-high $\mathrm{z}$-score of 47.63 belonged to

15 the TRY1 model trained by 986 data. The absolute values of 17 feature coefficients

16 ranged from 0.01 to 1.34 . The three top features in descending order of their absolute

17 values of weights were the count of nitrogen and oxygen atoms in the molecule $(-1.34$,

$1823.1 \%$ of all), the polar surface area $(1.01,17.4 \%$ of all), and the count of hydrogens

19 attached to nitrogen or oxygen atoms $(-0.518,8.9 \%$ of all). The last model examined 
1 was DRD3, which had the forth-high z-score value of 47.43 and was trained by 3,535

2 data. Its absolute values of 17 feature coefficients ranged from 0.01 to 1.25 . The three

3 top features in descending order of their absolute values of weights were the number

4 of non-hydrogen atoms in the molecule $(-1.25,23.4 \%$ of all $)$, the value of $\log \mathrm{P}(0.73$,

$513.8 \%$ of all), and the polar surface area $(0.69,13 \%$ of all).

6 Among the four models, three of them had polar surface area (PSA) as an

7 important feature, which is defined as the surface sum over all polar atoms or

8 molecules [53]. In general, bindings between proteins and drugs will be affected by

9 non-bonded interactions, which can be further divided into electrostatic interactions

10 and Van der Waals interactions. While many of the binding interactions depend a lot

11 on the formation of hydrogen bonds and salt bridges, the ligands with more PSA

12 might bring about stronger electrostatic interactions. Also, the PSA property is related

13 to the solvation of a molecule and will affect the inhibition ability of a drug to its

14 target protein. Therefore, PSA played an important role in binding strength prediction

15 and weighed higher in our models [54].

16 The other features with high weights were also associated with hydrogen atoms

17 and hydrogen bonds, including the number of hydrogen bond donors (HBD), the

18 count of nitrogen and oxygen atoms (HBA Lipinski), count of hydrogens attached to

19 nitrogen or oxygen atoms (HBD Lipinski), and the number of non-hydrogen atoms in 
1 the molecule. Unlike PSA, the weights, or coefficients, of these features differ in sign.

2 This is probably because that different target proteins may serve as donors or

3 acceptors of hydrogen bonds, while the number of donors/acceptors of drugs and the

4 binding sites of proteins also contribute to the interaction, leading to diverse

5 prediction pattern in different protein models.

6 Next, we tried to apply the similarity of feature coefficients between protein

7 models to drug repositioning. Among all 154,290 protein pairs, there were 37 pairs

8 with SCC values of coefficients higher than 0.5 among, and of which each of the 35

9 pairs was from the same protein family. Since proteins in a family typically have

10 similar structures, functions, and sequence similarity, it was reasonable for their

11 prediction models with similar feature weight ranking, that is, with higher SCC. Also,

12 for the proteins in the same family, most of the experimented drugs were the same,

13 limiting the efficacy of repositioning.

14 Thus, to further discover the capability of identifying the drug repositioning

15 between proteins from different family, we tried to study the other two protein pairs,

16 SYUA-TAU, and CY24B-WEE1. Since no research of common ligands to these two

17 proteins was found, this makes it difficult to demonstrate the association between

18 CYBB and WEE1. Additionally, the numbers of drug data used to train the models are

19 relatively small, which were 19 and 14 for CY24B and WEE1, respectively. The size 
1 of training sets might not be enough for models to approximate precise functions for

2 prediction, and lead to bias when comparing model coefficients. Consequently, we

3 focused on the protein pair, SYUA-TAU. The coefficients of the models of SYUA and

4 TAU had the second-high correlation among all the protein pairs, with an SCC value

5 of 0.86 . SYUA is a protein called alpha-synuclein encoded by the SNCA gene. It

6 mainly locates in the brain other than other tissues and is critical for brain function

7 [55]. And TAU is a protein named microtubule-associated protein tau, which is

8 encoded by gene $M A P T[56,57]$. TAU can stabilize microtubules and is abundant in

9 neurons of the central nervous system. Both proteins are associated with pathologies

10 and dementias of the nervous system like Parkinson's disease and Alzheimer's disease,

11 in which abnormal aggregations into amyloid of these proteins are often observed. In

12 these disorders, the alpha-synuclein protein and Tau protein form insoluble fibrils that

13 are characterized by Lewy bodies and neurofibrillary tangles respectively [58].

14 The SYUA model and TAU model were built by 45 and 46 drug data separately,

15 while the 45 drugs were the same and the TAU model had one more drug, FDDNP, in

16 the training dataset. FDDNP

17 (2-(1-\{6-[(2-[fluorine-18]fluoroethyl)(methyl)amino]-2-naphthyl \}-ethylidene)malono

18 nitril) is a positron emission tomography (PET) molecular imaging probe used to

19 visualize Alzheimer's disease pathology and its ability to detect amyloid in the brain is 
1 already proved. Due to the model similarity between the two proteins, FDDNP might

2 also have binding interaction with SYUA. Additionally, it has been reported that

3 FDDNP has a binding interaction with alpha-synuclein filaments in vitro and the $K_{i}$

4 value is $210 \mathrm{nM}$, while in our study the $K_{i}$ value of FDDNP binding to TAU was 256

5 nM [59]. The two close experimental $K_{i}$ values suggested that by comparing model

6 coefficients, we could find the proteins that may have similar binding patterns and

7 even share common ligands. However, FDDNP is majorly used as radiotracers of PET

8 for clinical diagnosis for Alzheimer's disease and seldom serves as direct medication

9 for treatments. This case demonstrated a promising application in drug repositioning,

10 though it is not the direct usage in treatments.

11 Our study so far focused on building single model for each protein and used only

12 drug structures and physicochemical properties as features. In the future, an advanced

13 goal is to incorporate features of protein and drug to construct a general model that

14 can predict the binding strength once given a protein-drug pair. Unlike the single

15 models that were restricted to the amount of experimental data of proteins, the general

16 model takes protein features like structures into account and is trained by the binding

17 information targeting all proteins, making it more feasible for prediction of rare

18 protein-drug instances. 


\section{Conclusions}

2 In this study, we applied a machine learning approach to predict the drug-protein

3 interaction and extended the result to construct a protein-protein network for drug

4 repositioning. We demonstrated that the models trained by more drug data had better

5 performance and less risk of overfitting when comparing the SCC values between

6 training and testing results. We further showed that our protein models were able to

7 recognize their own drugs from all the compounds in the dataset, which suggested that

8 the models could be applied for screening of candidate drugs. Also, by comparing

9 model coefficients of physicochemical properties, protein pair SYUA-TAU was found

10 to have alike biological role on GO term annotation and similar binding interactions

11 with shared drugs. When using protein $\mathrm{eBC}$ network based on proportionality for drug

12 repositioning, we found a potential target protein $\mathrm{AK} 1 \mathrm{C} 2$ of aspirin by investigating

13 the two-step neighbors around its known target PGH1. Briefly, our method can not

14 only be applied to drug repositioning by comparing protein models or searching the

15 protein-protein network, but also by predicting the binding strength directly once the

16 experimental data are sufficient to train the protein models. When given a drug with

17 structure and property information, we can get its binding strengths targeting different

18 proteins and find the one with the highest value as a new medication. Also given a

19 specific protein, we can use its model to predict all the drugs that have experimental 
1 data and find those with high returned values for alternative medication. As the

2 accumulation of experimental data and clinical research, we believe that the problem

3 raised by data size will be solved soon in the future and by using our method, the

4 efficiency and cost of drug development can be greatly improved.

$6 \quad$ List of abbreviations

7 SMD: small molecule drugs

8 FDA: Food and Drug Administration

9 SVM: support vector machine

10 SVR: support vector regression

11 SCC: Spearman correlation coefficient

12 eBC: edge betweenness centrality

13 MSE: mean-square error

14

15 Declarations

16 Ethics approval and consent to participate

17 Not applicable. 


\section{Consent for publication}

2 Not applicable.

4 The datasets used and analyzed in this study are available in the Binding DB website

5 (https://www.bindingdb.org); the ChEMBL repository (https://www.ebi.ac.uk/chembl);

6 and the Gene Ontology resource (http://geneontology.org/).

\section{7 - Competing interests}

8 The authors declare that they have no competing interests

9

10 This work was supported by Ministry of Science and Technology of Taiwan (MOST

11 104-2320-B-010-037- and MOST 107-2221-E-010-016-MY2); Institute of

12 Biomedical Informatics, National Yang-Ming University; and Department of Life

13 Sciences and Institute of Genome Sciences, National Yang-Ming University.

\section{Authors' contributions}

15 SYS and CCL directed this study. CCL conceived and designed this study. YTL

16 implemented the computational method and carried out the analysis. YTL drafted the 
1 manuscript. SYS and CCL revised the manuscript. All the authors read and approved

2 the final manuscript.

3

4 We thank Dr. Hsuan-Cheng Huang and Dr. Yu-Chao Wang in the Institute of

5 Biomedical Informatics, National Yang-Ming University provided invaluable

6 suggestions in improving this study.

8 Reference

9 1. Ciociola AA, Cohen LB, Kulkarni P, Gastroenterology FD-RMCotACo: How 10 drugs are developed and approved by the FDA: current process and future 11 directions. Am J Gastroenterol 2014, 109(5):620-623.

12 2. Elhassa GO: Drug Development: Stages of Drug Development. Journal of 13 Pharmacovigilance 2015, 03(03).

14 3. Zurdo J: Developability assessment as an early de-risking tool for 15 biopharmaceutical development. Pharmaceutical Bioprocessing 2013, 16 1(1):29-50.

17 4. DiMasi JA, Grabowski HG, Hansen RW: Innovation in the pharmaceutical 18 industry: New estimates of R\&D costs. J Health Econ 2016, 47:20-33.

19 5. DiMasi JA, Hansen RW, Grabowski HG: The price of innovation: new 20 estimates of drug development costs. Journal of Health Economics 2003, $21 \quad$ 22(2):151-185.

22 6. Vanhaelen Q: Computational Methods for Drug Repurposing; 2019.

23 7. Lysenko A, Sharma A, Boroevich KA, Tsunoda T: An integrative machine 24 learning approach for prediction of toxicity-related drug safety. Life Sci $25 \quad$ Alliance 2018, 1(6):e201800098.

26 8. Karimi M, Wu D, Wang Z, shen Y: DeepAffinity: Interpretable Deep Learning 27 of Compound Protein Affinity through Unified Recurrent and Convolutional $28 \quad$ Neural Networks. 2018. 
1 9. Riddick $G$, Song $H$, Ahn S, Walling J, Borges-Rivera D, Zhang W, Fine HA: Predicting in vitro drug sensitivity using Random Forests. Bioinformatics 2011, 27(2):220-224.

10. Agarwal S, Dugar D, Sengupta S: Ranking chemical structures for drug discovery: a new machine learning approach. J Chem Inf Model 2010, 50(5):716-731.

11. Napolitano F, Zhao Y, Moreira VM, Tagliaferri R, Kere J, D'Amato M, Greco D: Drug repositioning: a machine-learning approach through data integration. $J$ Cheminform 2013, 5(1):30.

12. Kim E, Choi AS, Nam H: Drug repositioning of herbal compounds via a machine-learning approach. BMC Bioinformatics 2019, 20(Suppl 10):247.

13. Li Z, Wang $Y$, Xie $Y$, Zhang L, Dai Z, Zou X: Predicting the binding affinities of compound-protein interactions by random forest using network topology features. Analytical Methods 2018, 10(34):4152-4161.

14. Chen X, Liu M, Gilson MK: BindingDB: a web-accessible molecular recognition database. Comb Chem High Throughput Screen 2001, 4(8):719-725.

15. Chen $X$, Lin $Y$, Liu M, Gilson MK: The Binding Database: data management and interface design. Bioinformatics 2002, 18(1):130-139.

16. Gilson MK, Liu T, Baitaluk M, Nicola G, Hwang L, Chong J: BindingDB in 2015: A public database for medicinal chemistry, computational chemistry and systems pharmacology. Nucleic Acids Res 2016, 44(D1):D1045-1053.

17. Liu T, Lin Y, Wen X, Jorissen RN, Gilson MK: BindingDB: a web-accessible database of experimentally determined protein-ligand binding affinities. Nucleic Acids Res 2007, 35(Database issue):D198-201.

18. Chen $X$, Lin Y, Gilson MK: The binding database: Overview and user's guide. Biopolymers 2001, 61(2):127-141.

19. Morley-Smith AC, Mills A, Jacobs S, Meyns B, Rega F, Simon AR, Pepper JR, Lyon AR, Thum $\mathrm{T}$ : Circulating microRNAs for predicting and monitoring response to mechanical circulatory support from a left ventricular assist device. European journal of heart failure 2014, 16(8):871-879.

20. McGregor MJ, Pallai PV: Clustering of Large Databases of Compounds:? Using the MDL "Keys" as Structural Descriptors. Journal of Chemical Information and Computer Sciences 1997, 37(3):443-448.

21. Durant JL, Leland BA, Henry DR, Nourse JG: Reoptimization of MDL keys for use in drug discovery. J Chem Inf Comput Sci 2002, 42(6):1273-1280. 
1 22. Gautam R, Seider WD: Computation of phase and chemical equilibrium: Part I. Local and constrained minima in Gibbs free energy. AlChE Journal 1979, 25(6):991-999.

23. Smola AJ, Schölkopf B: A tutorial on support vector regression. Statistics and Computing 2004, 14(3):199-222.

24. Gaulton A, Hersey A, Nowotka M, Bento AP, Chambers J, Mendez D, Mutowo P, Atkinson F, Bellis LJ, Cibrian-Uhalte E et al: The ChEMBL database in 2017. Nucleic Acids Res 2017, 45(D1):D945-D954.

25. Gaulton A, Bellis LJ, Bento AP, Chambers J, Davies M, Hersey A, Light $Y$, McGlinchey S, Michalovich D, Al-Lazikani B et al: ChEMBL: a large-scale bioactivity database for drug discovery. Nucleic Acids Res 2012, 40(Database issue):D1100-1107.

26. Krasner J: Drug-Protein Interaction. Pediatric Clinics of North America 1972, 19(1):51-63.

27. Akoglu H: User's guide to correlation coefficients. Turk J Emerg Med 2018, 18(3):91-93.

28. Spearman C: The Proof and Measurement of Association between Two Things. The American Journal of Psychology 1987, 100(3/4):441-471.

29. Hauke J, Kossowski T: Comparison of Values of Pearson's and Spearman's Correlation Coefficients on the Same Sets of Data. Quaestiones Geographicae 2011, 30(2):87-93.

30. Fieller EC, Hartley HO, Pearson ES: Tests for Rank Correlation Coefficients. I. Biometrika 1957, 44(3/4):470-481.

31. Erb I, Notredame C: How should we measure proportionality on relative gene expression data? Theory Biosci 2016, 135(1-2):21-36.

32. Lovell D, Pawlowsky-Glahn V, Egozcue JJ, Marguerat S, Bahler J: Proportionality: a valid alternative to correlation for relative data. PLOS Comput Biol 2015, 11(3):e1004075.

33. Quinn TP, Richardson MF, Lovell D, Crowley TM: propr: An R-package for Identifying Proportionally Abundant Features Using Compositional Data Analysis. Sci Rep 2017, 7(1):16252.

34. Girvan $M$, Newman ME: Community structure in social and biological networks. Proc Natl Acad Sci U S A 2002, 99(12):7821-7826.

35. Silverman LS, Caldwell JP, Greenlee WJ, Kiselgof E, Matasi JJ, Tulshian DB, Arik $\mathrm{L}$, Foster $\mathrm{C}$, Bertorelli $\mathrm{R}$, Monopoli $\mathrm{A}$ et al: $3 \mathrm{H}-[1,2,4]$-Triazolo[5,1-i]purin-5-amine derivatives as adenosine A2A antagonists. Bioorg Med Chem Lett 2007, 17(6):1659-1662. 
1 36. Albizu L, Holloway T, Gonzalez-Maeso J, Sealfon SC: Functional crosstalk and heteromerization of serotonin $5-\mathrm{HT} 2 \mathrm{~A}$ and dopamine D2 receptors. Neuropharmacology 2011, 61(4):770-777.

37. Wei $Y$, Pattingre $S$, Sinha $S$, Bassik $M$, Levine $B$ : JNK1-mediated phosphorylation of $\mathrm{Bcl}-2$ regulates starvation-induced autophagy. $\mathrm{Mol}$ Cell 2008, 30(6):678-688.

38. Bruey JM, Bruey-Sedano N, Luciano F, Zhai D, Balpai R, Xu C, Kress CL, Bailly-Maitre $\mathrm{B}$, Li $\mathrm{X}$, Osterman $\mathrm{A}$ et al: $\mathrm{BCl}-\mathbf{2}$ and $\mathrm{BCl}-\mathrm{XL}$ regulate proinflammatory caspase-1 activation by interaction with NALP1. Cell 2007, 129(1):45-56.

39. Wu XY, Zhang CX, Deng LC, Xiao J, Yuan X, Zhang B, Hou ZB, Sheng ZH, Sun L, Jiang QC et al: Overexpressed D2 Dopamine Receptor Inhibits Non-Small Cell Lung Cancer Progression through Inhibiting NF-kappaB Signaling Pathway. Cell Physiol Biochem 2018, 48(6):2258-2272.

40. Wu DM, Wang S, Wen X, Han XR, Wang YJ, Shen M, Fan SH, Zhuang J, Zhang $Z F$, Shan $Q$ et al: Inhibition of microRNA-200a Upregulates the Expression of Striatal Dopamine Receptor D2 to Repress Apoptosis of Striatum via the CAMP/PKA Signaling Pathway in Rats with Parkinson's Disease. Cell Physiol Biochem 2018, 51(4):1600-1615.

41. Stam NJ, Van Huizen F, Van Alebeek C, Dijkema R, Tonnaer JADM, Olijve W: Genomic organization, coding sequence and functional expression of human 5-HT2 and 5-HT1A receptor genes. European Journal of Pharmacology: Molecular Pharmacology 1992, 227(2):153-162.

42. Cussac D, Boutet-Robinet E, Ailhaud MC, Newman-Tancredi A, Martel JC, Danty $\mathrm{N}$, Rauly-Lestienne I: Agonist-directed trafficking of signalling at serotonin 5-HT2A, 5-HT2B and 5-HT2C-VSV receptors mediated Gq/11 activation and calcium mobilisation in CHO cells. Eur J Pharmacol 2008, 594(1-3):32-38.

43. Villar VA, Jones JE, Armando I, Palmes-Saloma C, Yu P, Pascua AM, Keever L, Arnaldo FB, Wang Z, Luo $Y$ et al: G protein-coupled receptor kinase 4 (GRK4) regulates the phosphorylation and function of the dopamine D3 receptor. $J$ Biol Chem 2009, 284(32):21425-21434.

44. Patrignani P, Patrono C: Aspirin and Cancer. J Am Coll Cardiol 2016, 68(9):967-976.

45. Warner TD, Mitchell JA: Cyclooxygenase-3 (COX-3): filling in the gaps toward a COX continuum? Proc Natl Acad Sci U S A 2002, 99(21):13371-13373. 
1 46. Seshasai SR, Wijesuriya S, Sivakumaran R, Nethercott S, Erqou S, Sattar N, Ray KK: Effect of aspirin on vascular and nonvascular outcomes: meta-analysis of randomized controlled trials. Arch Intern Med 2012, 172(3):209-216.

47. Penning TM, Burczynski ME, Jez JM, Hung CF, Lin HK, Ma $H$, Moore $M$, Palackal N, Ratnam K: Human 3alpha-hydroxysteroid dehydrogenase isoforms (AKR1C1-AKR1C4) of the aldo-keto reductase superfamily: functional plasticity and tissue distribution reveals roles in the inactivation and formation of male and female sex hormones. Biochem J 2000, 351(Pt 1):67-77.

48. Dufort I, Rheault $P$, Huang XF, Soucy $P$, Luu-The V: Characteristics of a highly labile human type 5 17beta-hydroxysteroid dehydrogenase. Endocrinology 1999, 140(2):568-574.

49. Ji Q, Aoyama C, Nien YD, Liu PI, Chen PK, Chang L, Stanczyk FZ, Stolz A: Selective loss of AKR1C1 and AKR1C2 in breast cancer and their potential effect on progesterone signaling. Cancer Res 2004, 64(20):7610-7617.

50. Ji Q, Chang L, VanDenBerg D, Stanczyk FZ, Stolz A: Selective reduction of AKR1C2 in prostate cancer and its role in DHT metabolism. Prostate 2003, 54(4):275-289.

51. Tai H-L, Lin T-S, Huang H-H, Lin T-Y, Chou M-C, Chiou S-H, Chow K-C: Overexpression of aldo-keto reductase $1 \mathrm{C} 2$ as a high-risk factor in bladder cancer. Oncology Reports 2007.

52. Byrns MC, Penning TM: Type 5 17beta-hydroxysteroid dehydrogenase/prostaglandin F synthase (AKR1C3): role in breast cancer and inhibition by non-steroidal anti-inflammatory drug analogs. Chem Biol Interact 2009, 178(1-3):221-227.

53. Ertl P, Rohde B, Selzer P: Fast calculation of molecular polar surface area as a sum of fragment-based contributions and its application to the prediction of drug transport properties. J Med Chem 2000, 43(20):3714-3717.

54. Pajouhesh H, Lenz GR: Medicinal chemical properties of successful central nervous system drugs. NeuroRx 2005, 2(4):541-553.

55. Stefanis L: alpha-Synuclein in Parkinson's disease. Cold Spring Harb Perspect Med 2012, 2(2):a009399.

56. Caillet-Boudin ML, Buee L, Sergeant N, Lefebvre B: Regulation of human MAPT gene expression. Mol Neurodegener 2015, 10:28.

57. Weingarten MD, Lockwood AH, Hwo SY, Kirschner MW: A protein factor essential for microtubule assembly. Proc Natl Acad Sci U S A 1975, 72(5):1858-1862. 
1 58. Lockhart A: Imaging Alzheimer's disease pathology: one target, many ligands.

21 for proteins. Blue and red bars showed the proteins with experimentally-validated

$$
\text { Drug Discov Today 2006, 11(23-24):1093-1099. }
$$

59. Ye L, Velasco A, Fraser G, Beach TG, Sue L, Osredkar T, Libri V, Spillantini MG, Goedert $M$, Lockhart $A$ : In vitro high affinity alpha-synuclein binding sites for the amyloid imaging agent PIB are not matched by binding to Lewy bodies in postmortem human brain. J Neurochem 2008, 105(4):1428-1437.

\section{Figure Legends}

\section{Figure 1. Performance of the prediction model from AA2AR, DHI, and AL5AP}

protein. a. The distribution of SCCs between experimental and predicted binding

affinity from trained (black) and tested (red) models of AA2AR, DHI1, and AL5AP.

The green arrow indicated the SCC of the complete dataset. b. Distributions of experimental (black) and predicted (red) binding affinity of drugs to AA2AR, DHI1, and AL5AP. c. Number of experimentally-validated drugs of AA2AR, DHI1, and AL5AP in ascending rankings of predicted binding affinity. The red and blue bars showed the experimentally-validated drugs in the corresponding ranking are significantly over- and under-represented, respectively (Fisher's exact test, $p$-value < $0.05)$.

\section{Figure 2. The association between number of experimentally-validated drugs and}

model performance. a. Distribution of the number of experimentally-validated drugs 
1 numbers and prediction SCC transformed z-score of models. c. Negative correlation

2 between the drug number and standard deviation of SCC. The standard deviation was

3 calculated from the SCCs between the experimentally-validated and predicted binding

4 affinity from 1,000 training model. d. Association between the drug numbers and

5 prediction stability. The prediction stability here is estimated by the difference of SCC

6 between the complete dataset and training set. (E) Average SCC z-score of models

7 predicting drugs of other proteins. Most of the models were unsuitable to predict the

8 binding interaction targeting proteins other than themselves.

9 Figure 3. The SCC of model coefficients and the mean drug number of model

10 pairs. For each protein pair, the SCC of model coefficients and average drug number

11 were calculated. The green line indicated where the smallest significantly positive

12 SCC (value $\geqq 0.15, p$-value $<0.05)$.

13 Figure 4. The functional similarity of the shortest paths in the protein similarity

14 network. a. The original network based on protein proportionalities. b. The auxiliary

15 eBC network of the 556 proteins. For the primeval network, the proteins were

16 fully-connected and could not be interpreted with corresponding biological

17 similarities. After removing connections with low edge betweenness centrality, the

18 network had positive correlation with GO term similarities and showed noticeable

19 structure. c. The protein pairs with a two-step distance had the highest GO similarity 
bioRxiv preprint doi: https://doi.org/10.1101/2020.07.29.218826; this version posted July 29, 2020. The copyright holder for this preprint (which was not certified by peer review) is the author/funder. All rights reserved. No reuse allowed without permission.

1 among pairs with each shortest paths. This implied that the indirect connections

2 between proteins could better reflect the biological similarities.

3

4 
A

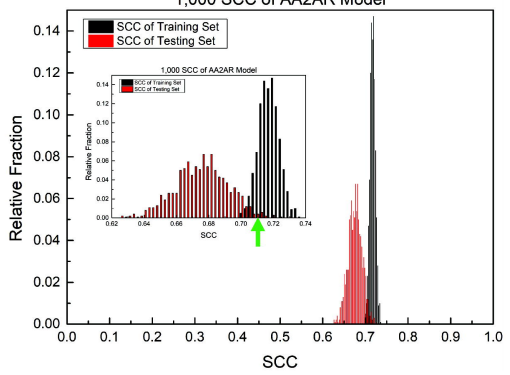

B

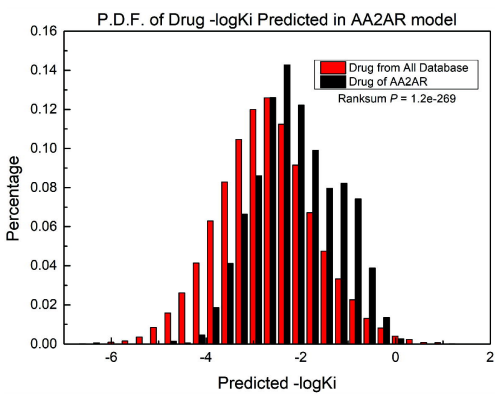

C

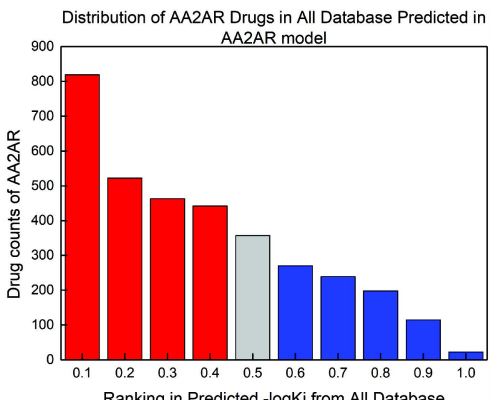

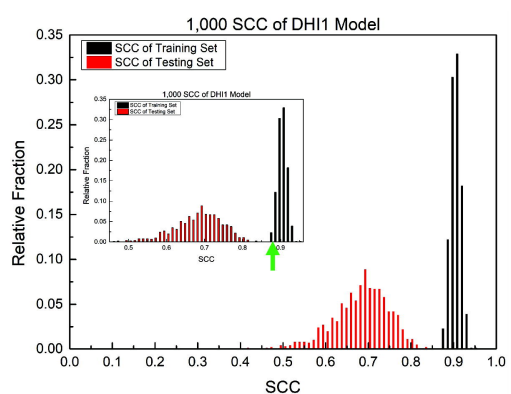
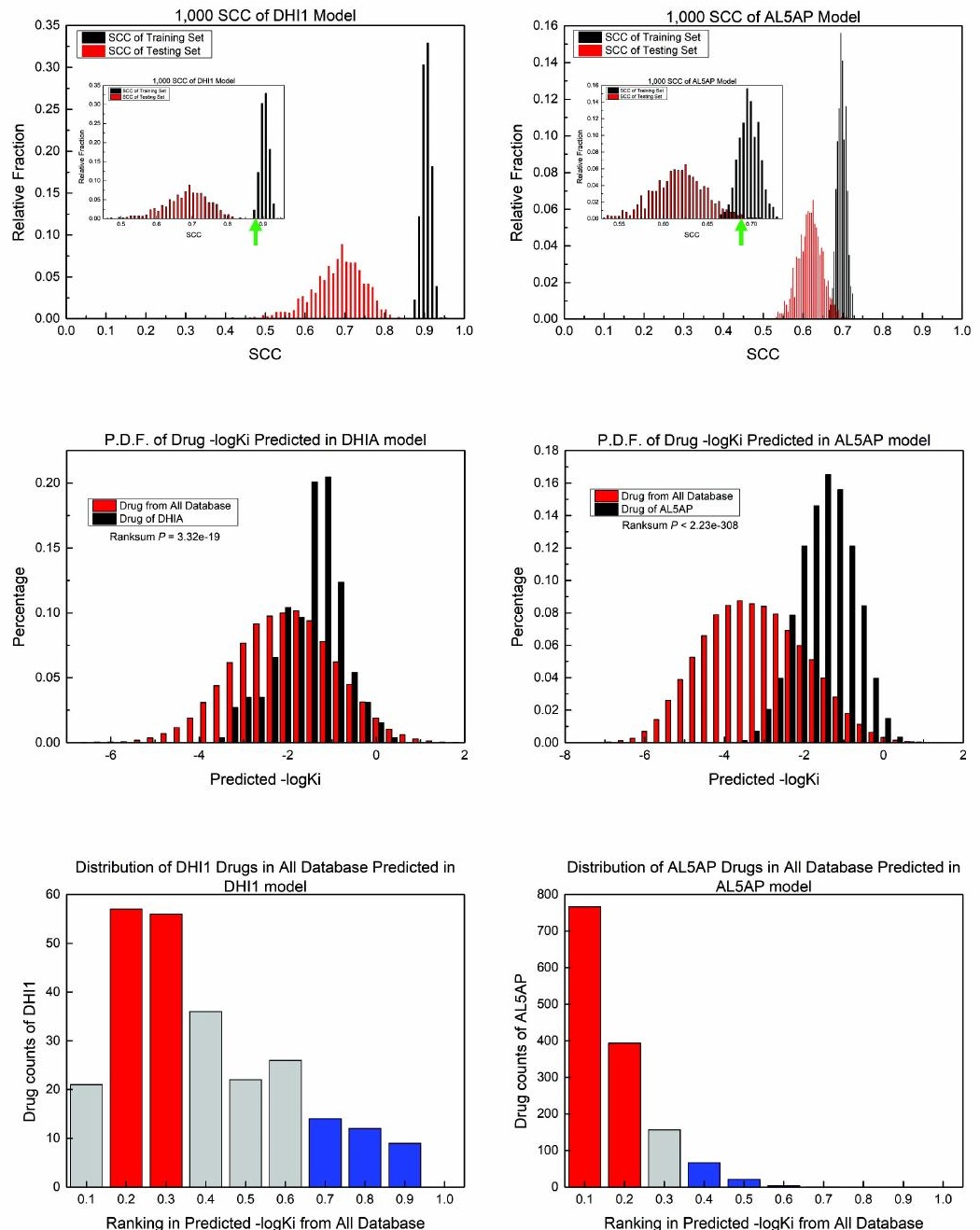
